Supporting Information for

\title{
Synthesis of Benzo[c]thiophenes by Rhodium(III)-Catalyzed
}

\section{Dehydrogenative Annulation}

Keita Fukuzumi ${ }^{\dagger}$ Yuto Unoh, ${ }^{\dagger}$ Yuji Nishii, ${ }^{\ddagger}$ Tetsuya Satoh, ${ }^{\S}$ Koji Hirano, ${ }^{\dagger}$ Masahiro Miura ${ }^{*},{ }^{\dagger}$

$\dagger$ Department of Applied Chemistry and $\star$ Frontier Research Base for Global Young Researchers, Graduate School of Engineering, Osaka University, Suita, Osaka 565-0871, Japan

$\S$ Department of Chemistry, Graduate School of Science, Osaka City University, 3-3-138 Sugimoto, Sumiyoshi-ku, Osaka 558-8585, Japan

E-mail:miura@chem.eng.osaka-u.ac.jp

\section{Contents}

I. VT-NMR and X-ray Analyses for Structural Determination of 3aa 2

II. $\quad{ }^{1} \mathrm{H}$ and ${ }^{13} \mathrm{C}$ NMR Spectra 3 


\section{VT-NMR and X-ray Analyses for Structural Determination of 3aa}

The annulation product 3aa showed broadened signals on NMR spectra due to the conformational isomerism of pyrrolidine 5-membered ring and slow amide bond rotation (amide rotamers). $\quad{ }^{1} \mathrm{H}$ NMR measurement at variable temperature displayed a reversible spectral change; however, reliable information of integration values and coupling constants were not obtained (Figure S1). Finally, the molecular structure of 3aa was determined by X-ray crystallography (Figure S2).
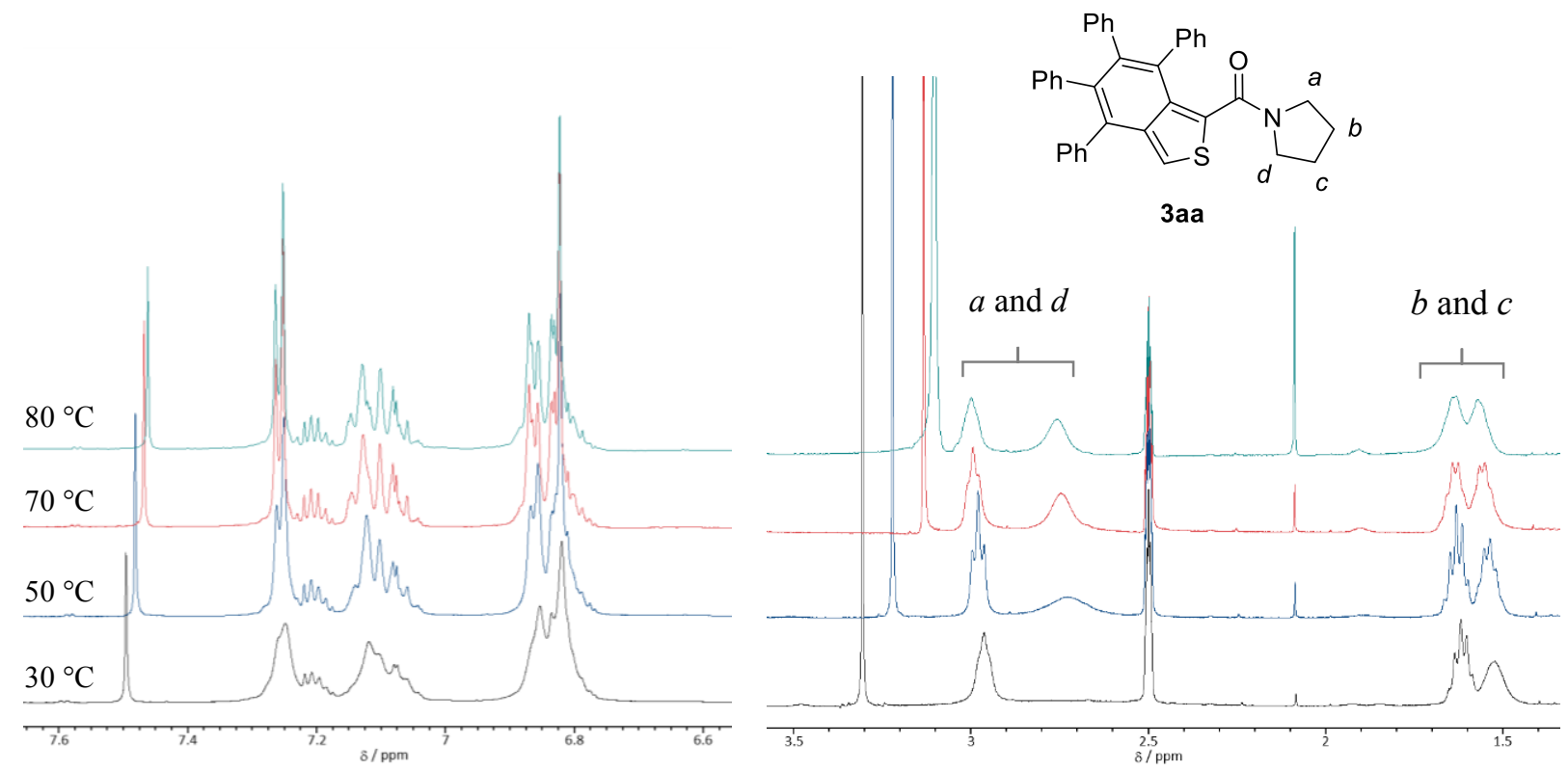

Figure S1. VT- ${ }^{1} \mathrm{H}$ NMR spectra of 3aa in DMSO- $d_{6}$. Spectra from $3.5 \mathrm{ppm}$ to $6.6 \mathrm{ppm}$ were omitted for clarity because no peak was observed in this region.

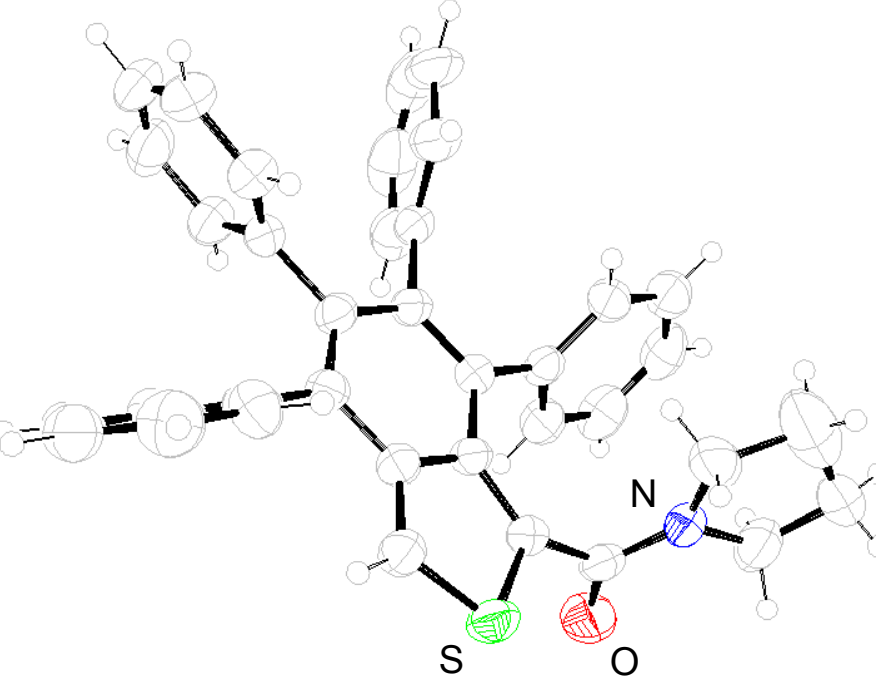

Figure S2. ORTEP drawing of 3aa with 50\% thermal ellipsoid (CCDC 1442267). 


\section{II. ${ }^{1} \mathrm{H}$ and ${ }^{13} \mathrm{C}$ NMR Spectra}

Pyrrolidin-1-yl(4,5,6,7-tetraphenylbenzo[c]thiophen-1-yl)methanone (3aa in $\mathrm{CDCl}_{3}$ )
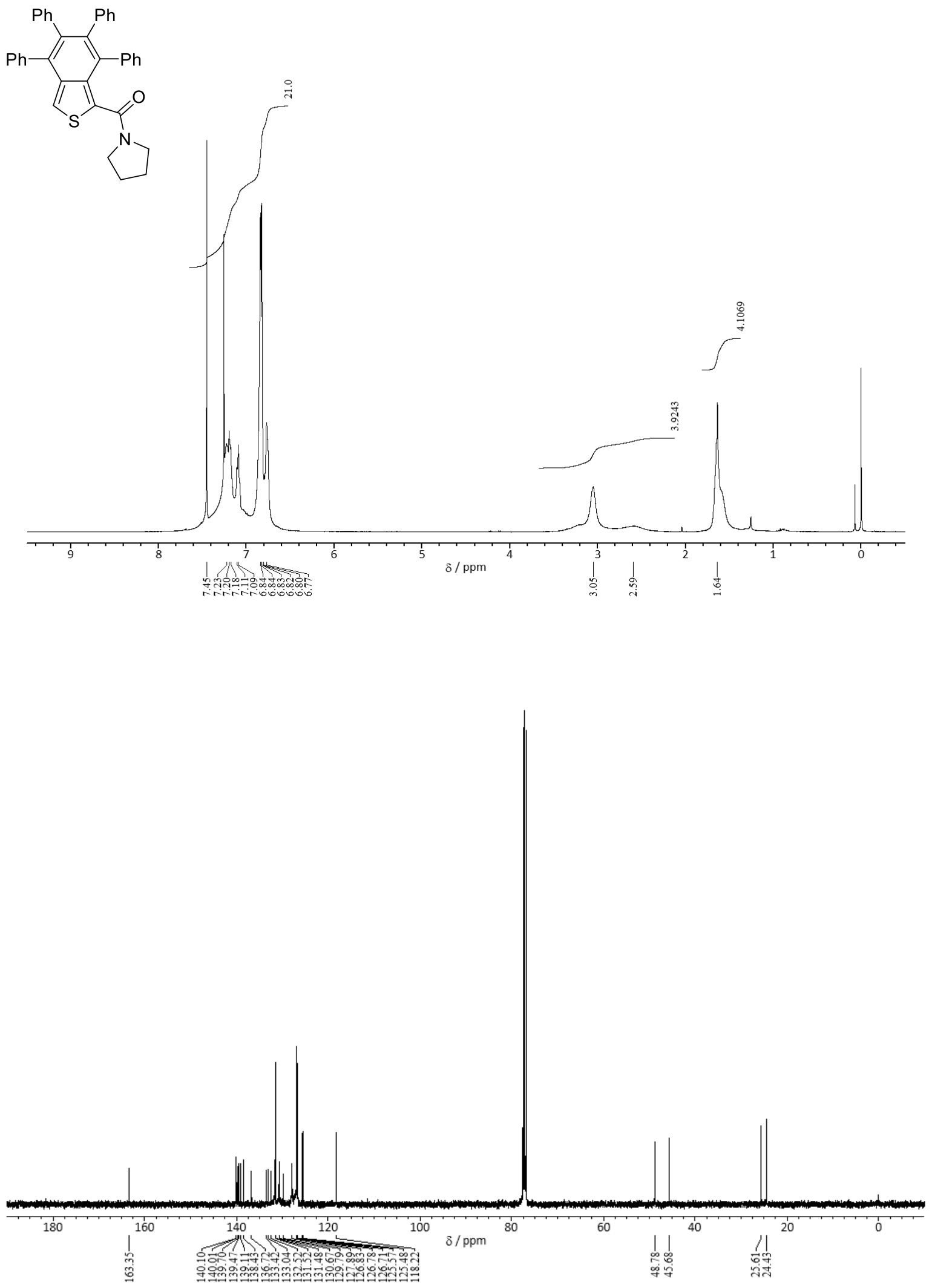
Pyrrolidin-1-yl(4,5,6,7-tetraphenylbenzo[c]thiophen-1-yl)methanone (3aa in DMSO- $d_{6}$ )
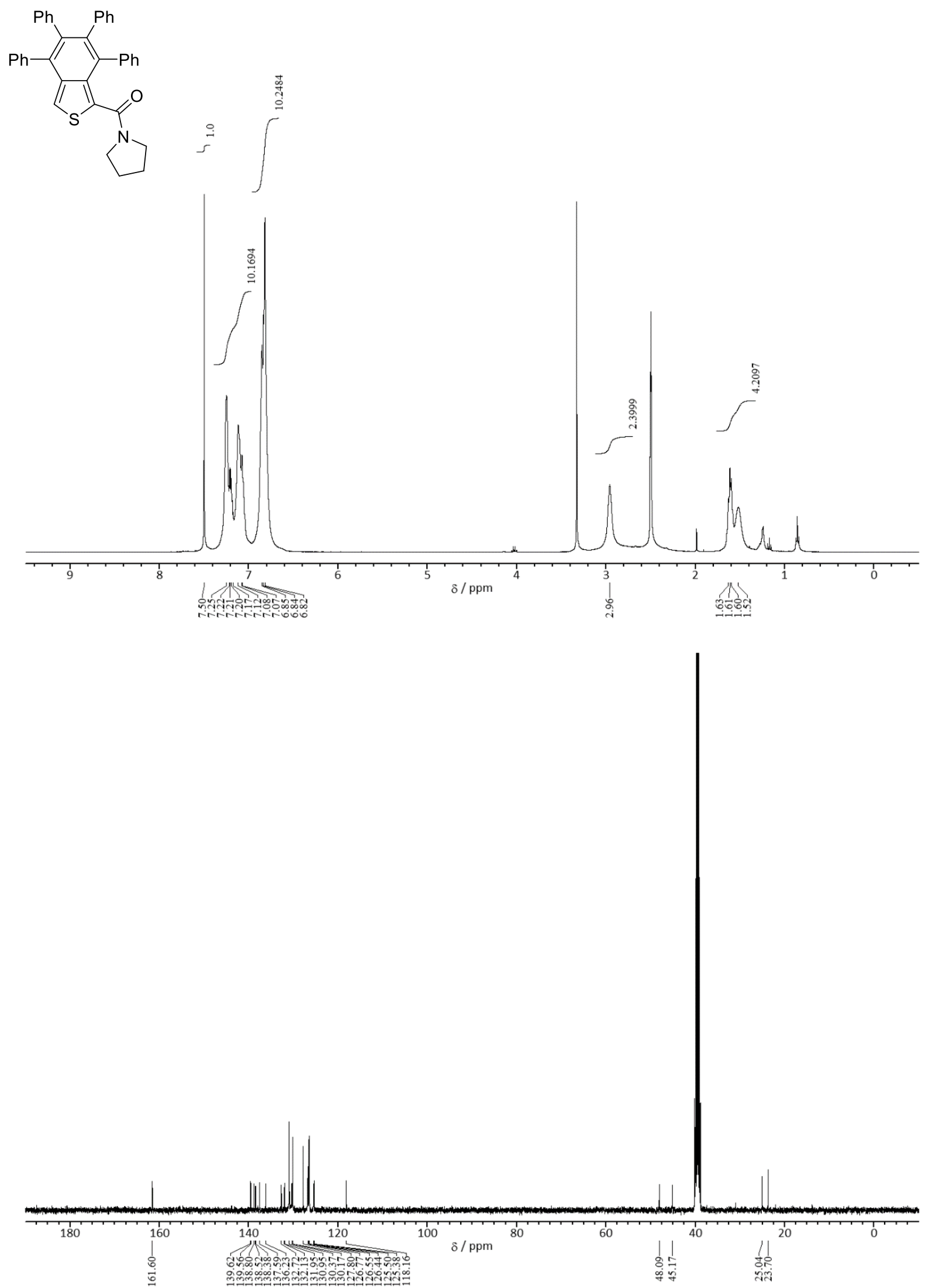
$N, N$-Diisopropyl-4,5,6,7-tetraphenylbenzo[c]thiophene-1-carboxamide (3ba)<smiles>CC(C)N(C(=O)c1scc2c(-c3ccccc3)c(-c3ccccc3)c(-c3ccccc3)c(-c3ccccc3)c12)C(C)C</smiles>
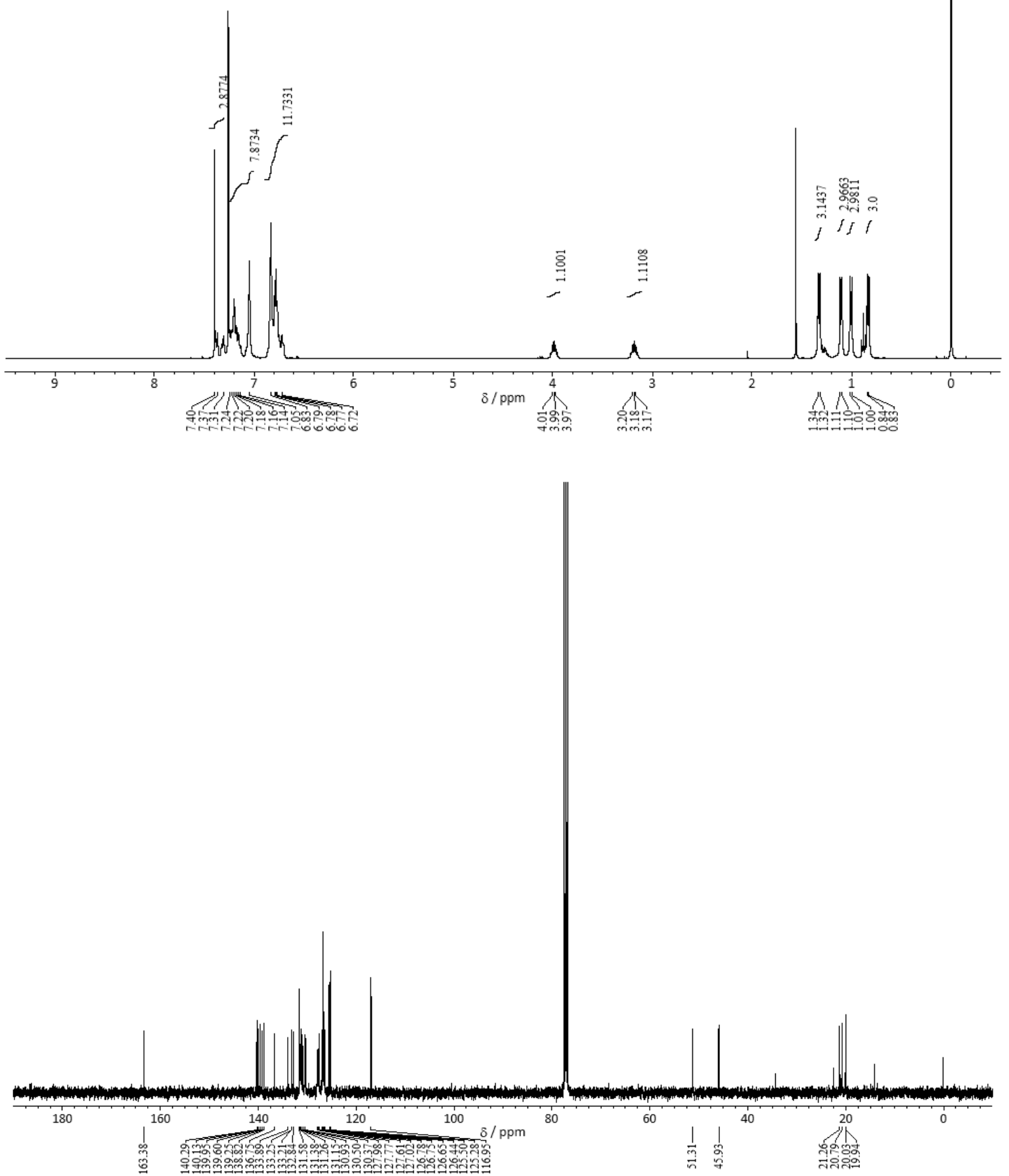
$N, N$-Diethyl-4,5,6,7-tetraphenylbenzo[c]thiophene-1-carboxamide (3ca)
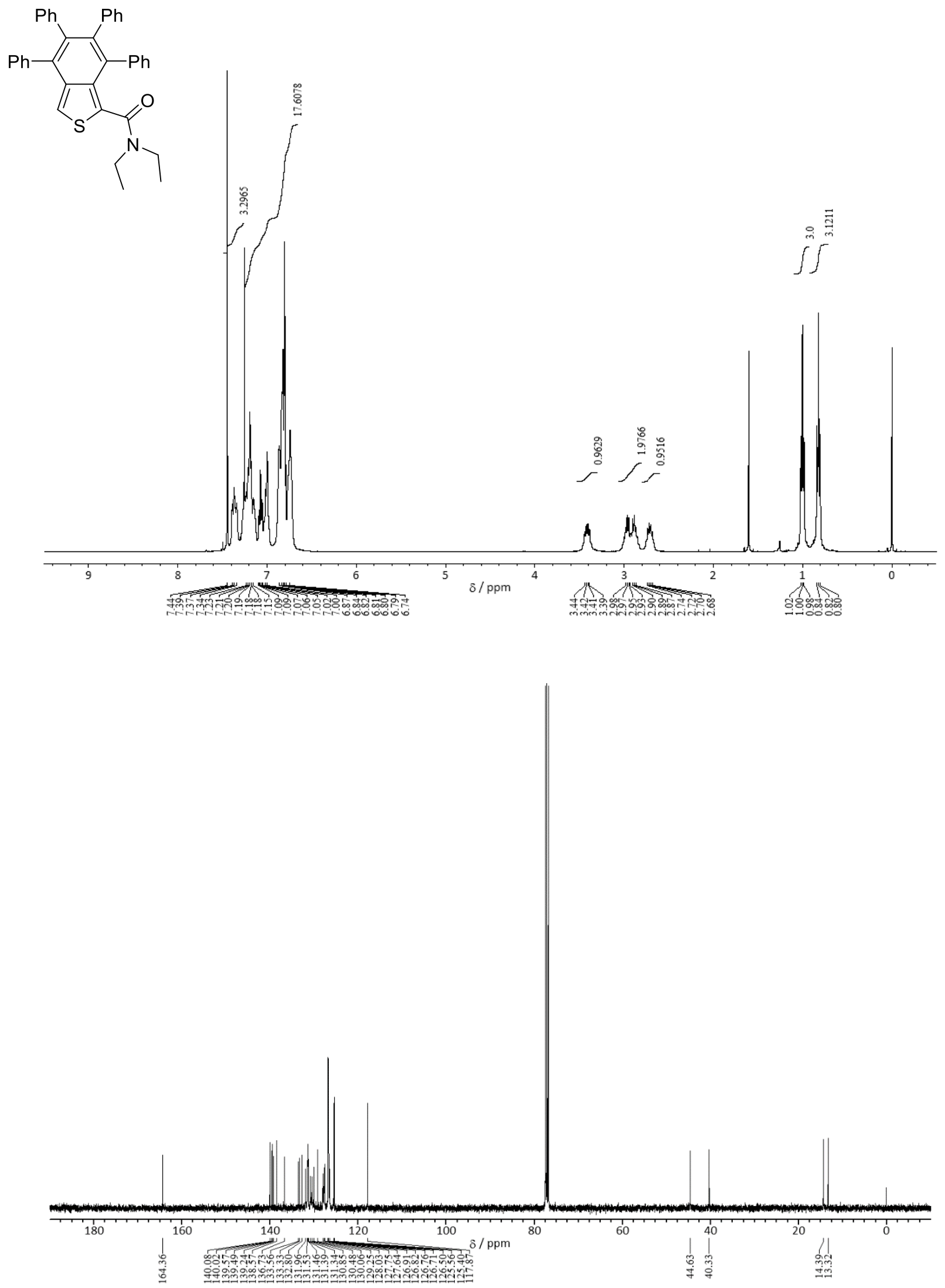
$N, N$-Dimethyl-4,5,6,7-tetraphenylbenzo[c]thiophene-1-carboxamide (3da)<smiles>CN(C)C(=O)c1scc2c(-c3ccccc3)c(-c3ccccc3)c(-c3ccccc3)c(-c3ccccc3)c12</smiles>

$\int \frac{3}{3}$
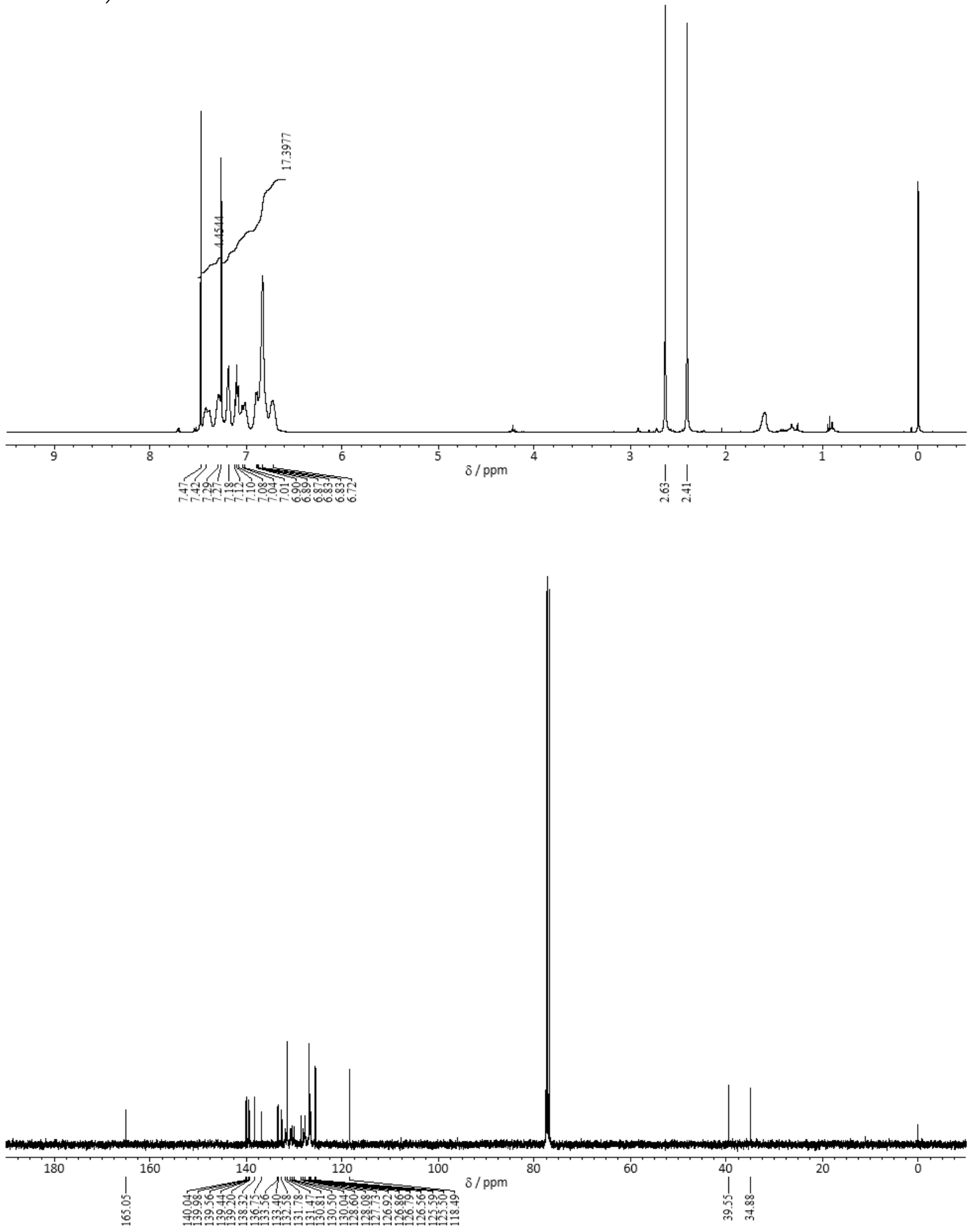
$N, N$-Diethyl-4,5,6,7-tetra- $p$-tolylbenzo[c] thiophene-1-carboxamide (3cb)
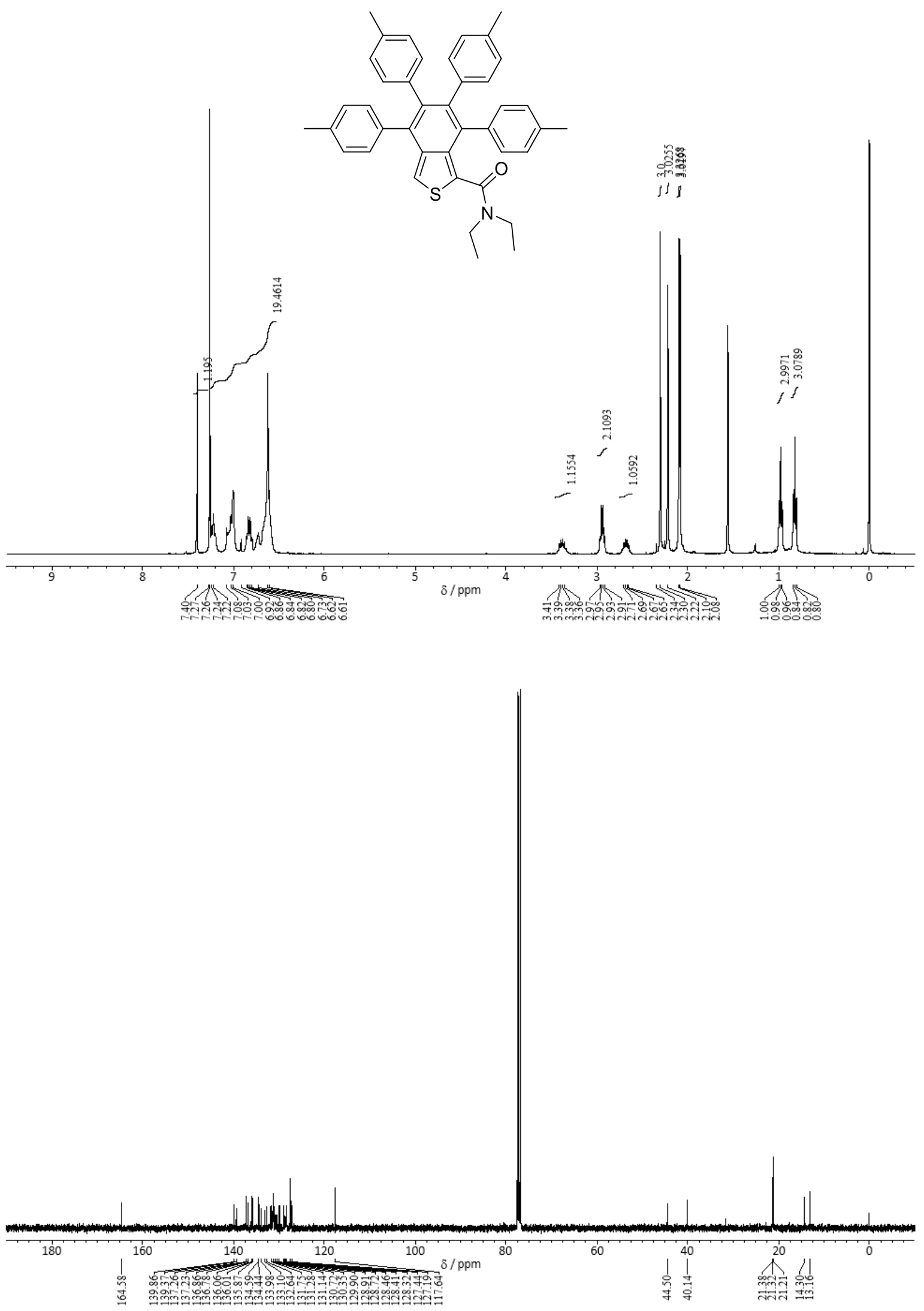

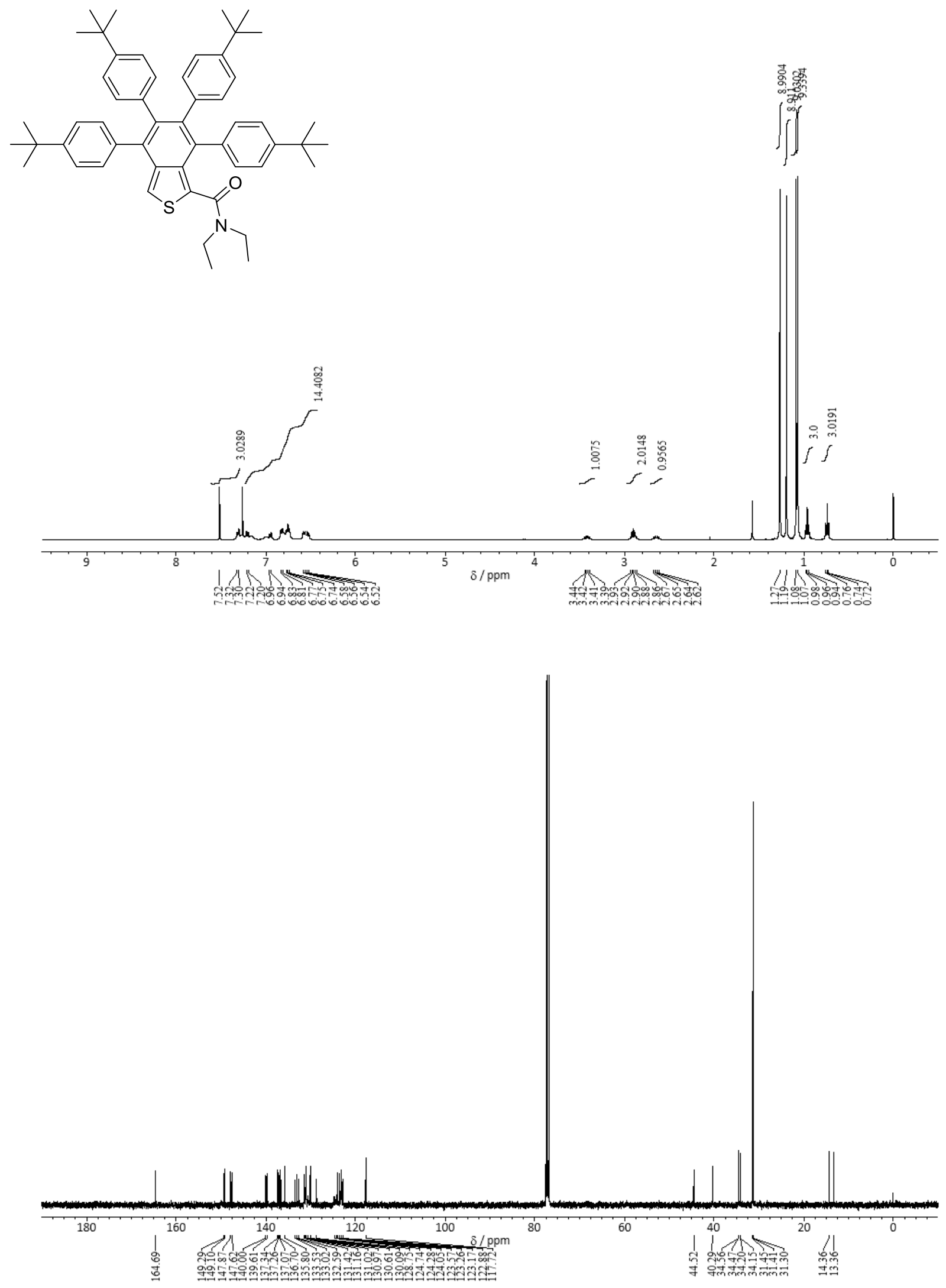
$N, N$-Diethyl-4,5,6,7-tetrakis(4-fluorophenyl)benzo[c]thiophene-1-carboxamide (3cd)
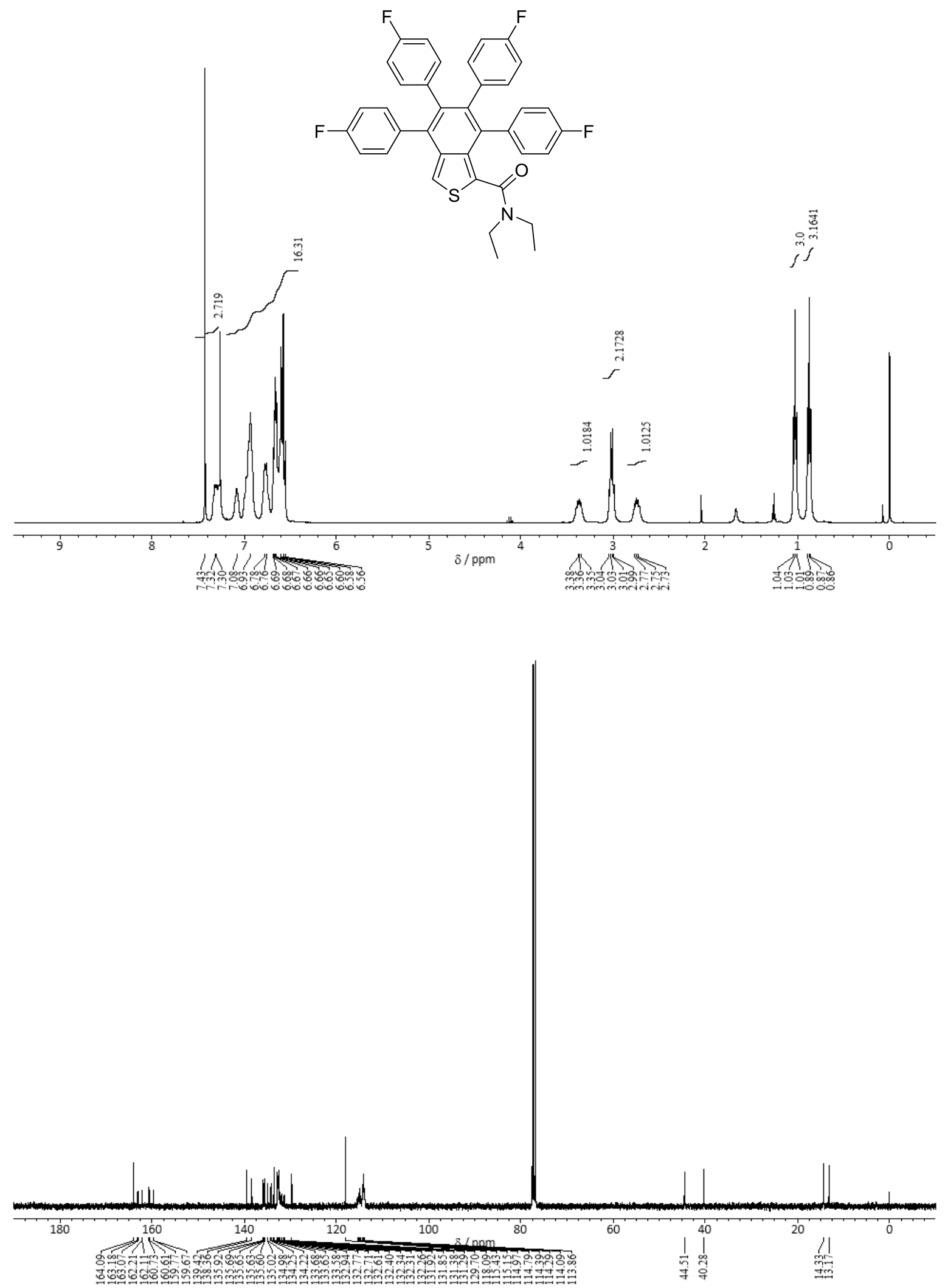
${ }^{13} \mathrm{C}\left\{{ }^{1} \mathrm{H},{ }^{19} \mathrm{~F}\right\}$ NMR spectrum for $\mathbf{3 c d}$

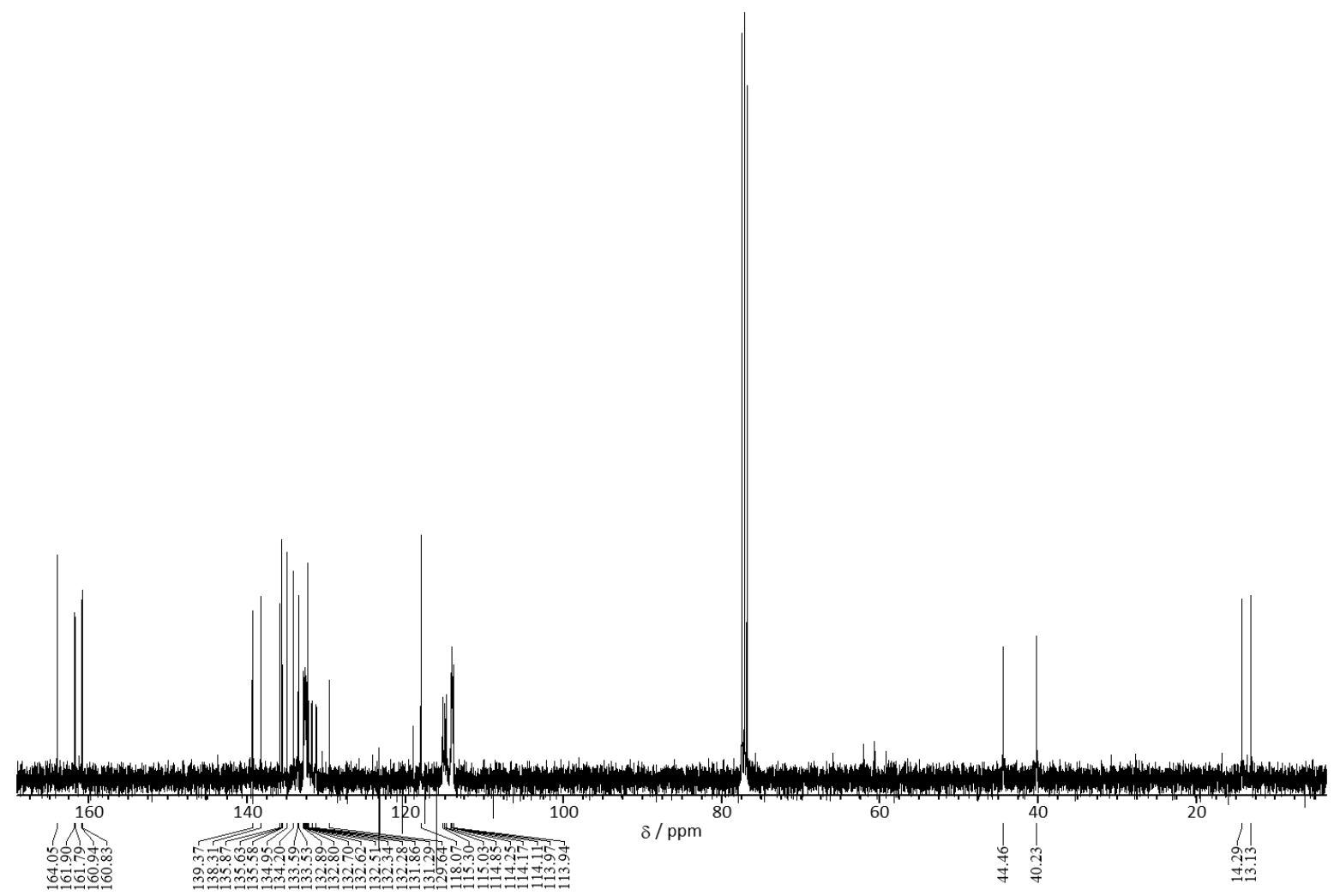


$N, N$-Diethyl-4,5,6,7-tetrakis(4-(trifluoromethyl)phenyl)benzo[c]thiophene-1-carboxamide (3ce)
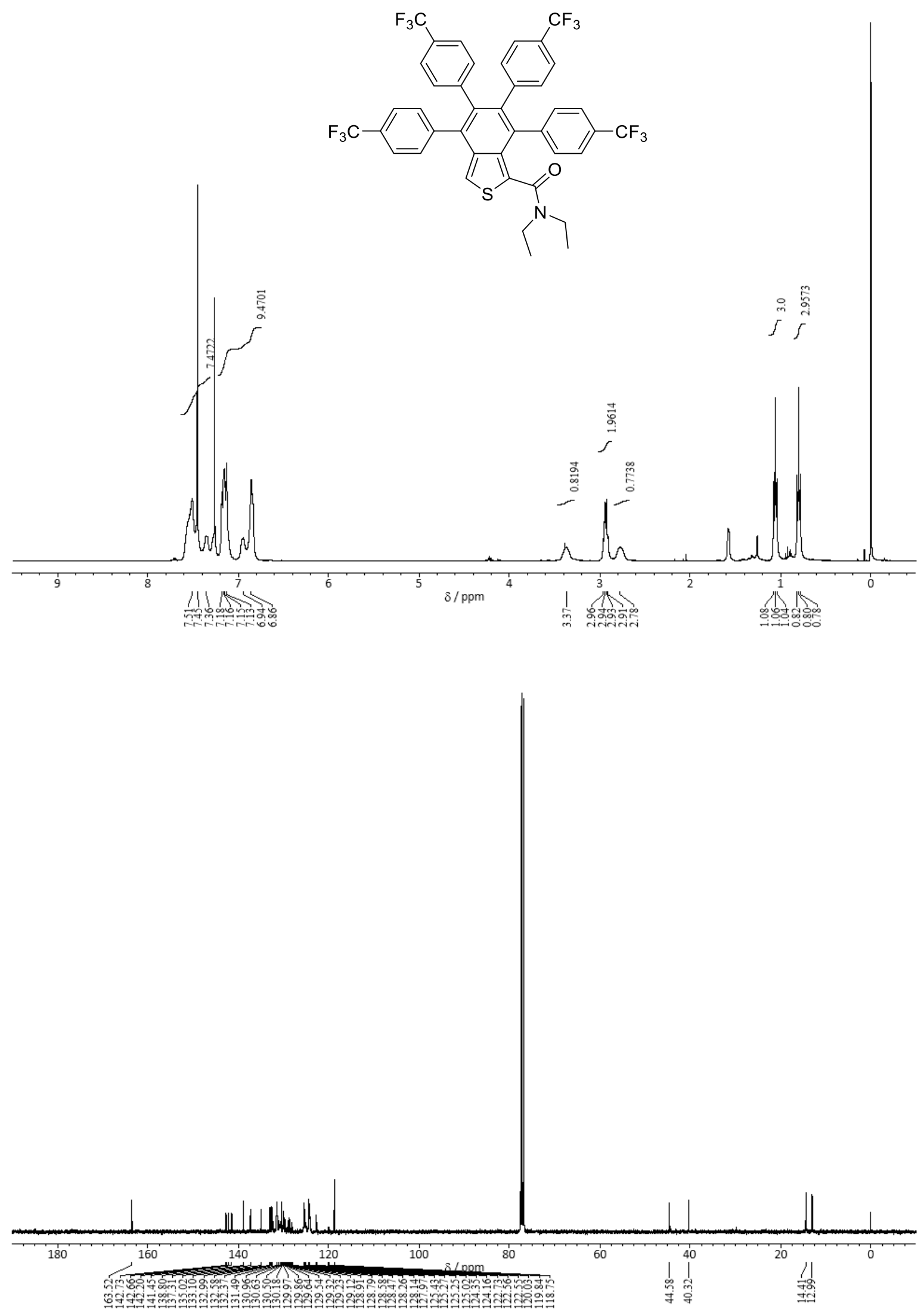
${ }^{13} \mathrm{C}\left\{{ }^{1} \mathrm{H},{ }^{19} \mathrm{~F}\right\}$ NMR spectrum for 3 ce

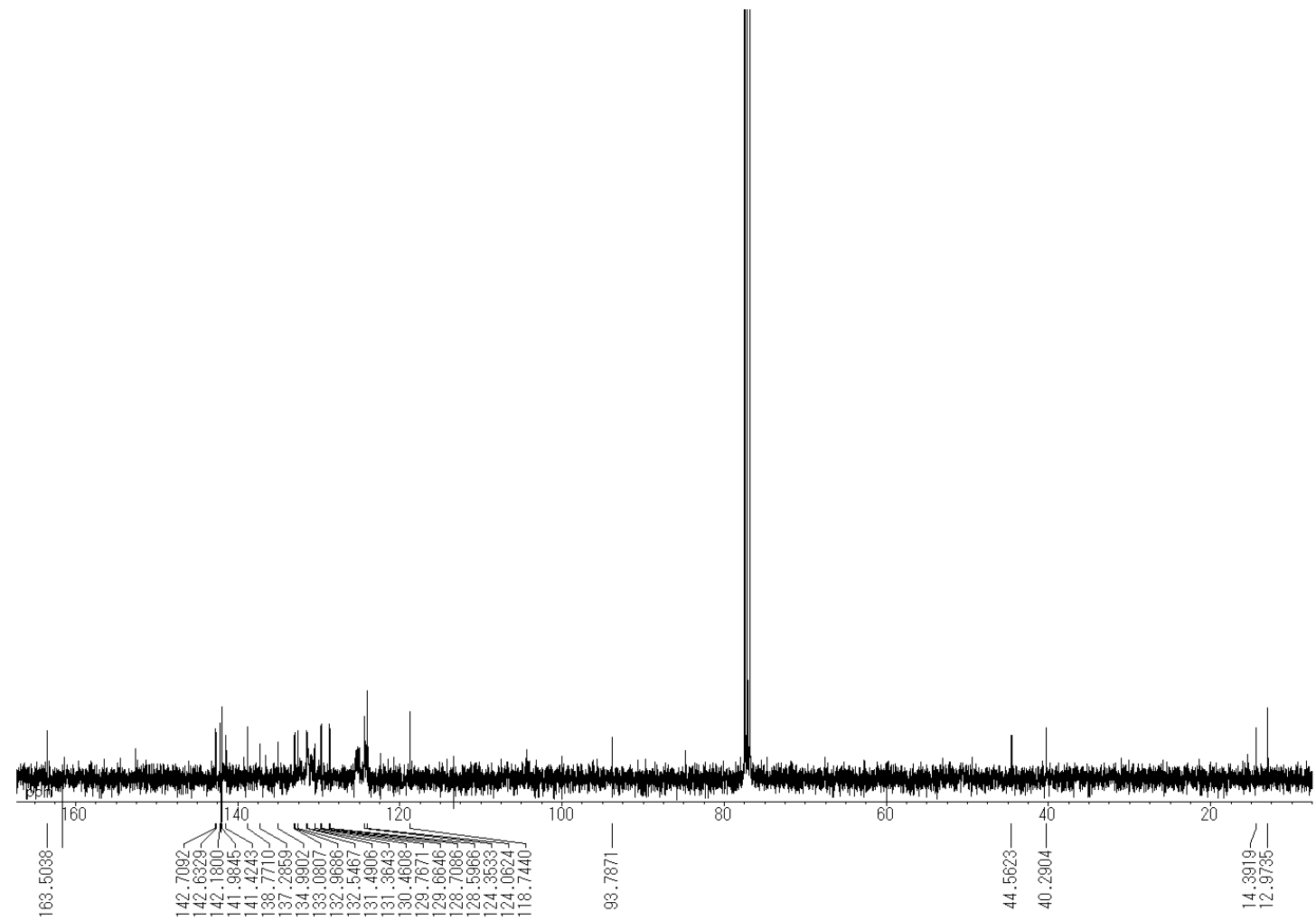


Tetraethyl 4,4',4",4"'-(1-(diethylcarbamoyl)benzo[c]thiophene-4,5,6,7-tetrayl)tetrabenzoate (3cf)
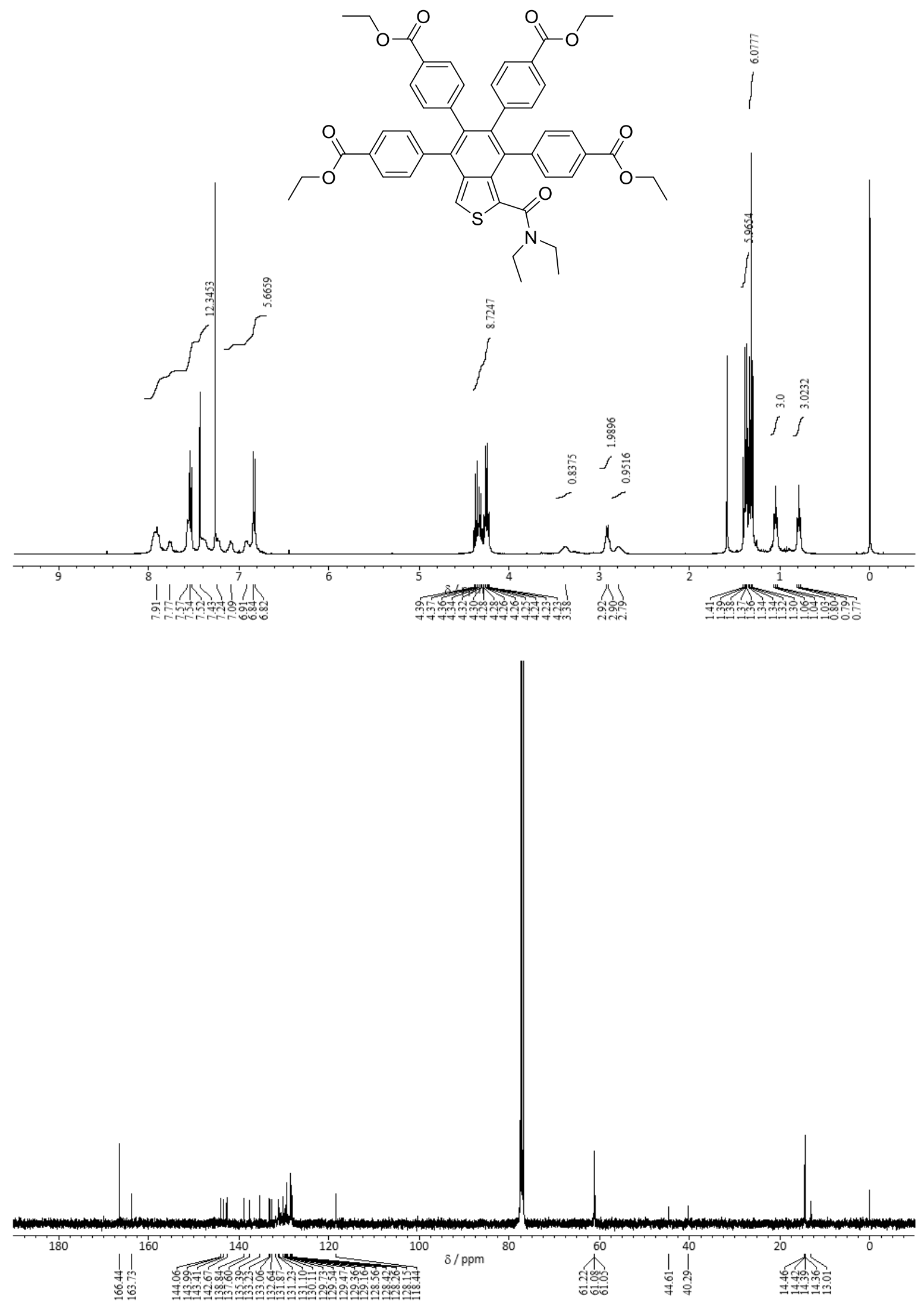
$N, N$-Diethyl-4,5,6,7-tetrakis(4-methoxyphenyl)benzo[c]thiophene-1-carboxamide (3cg)
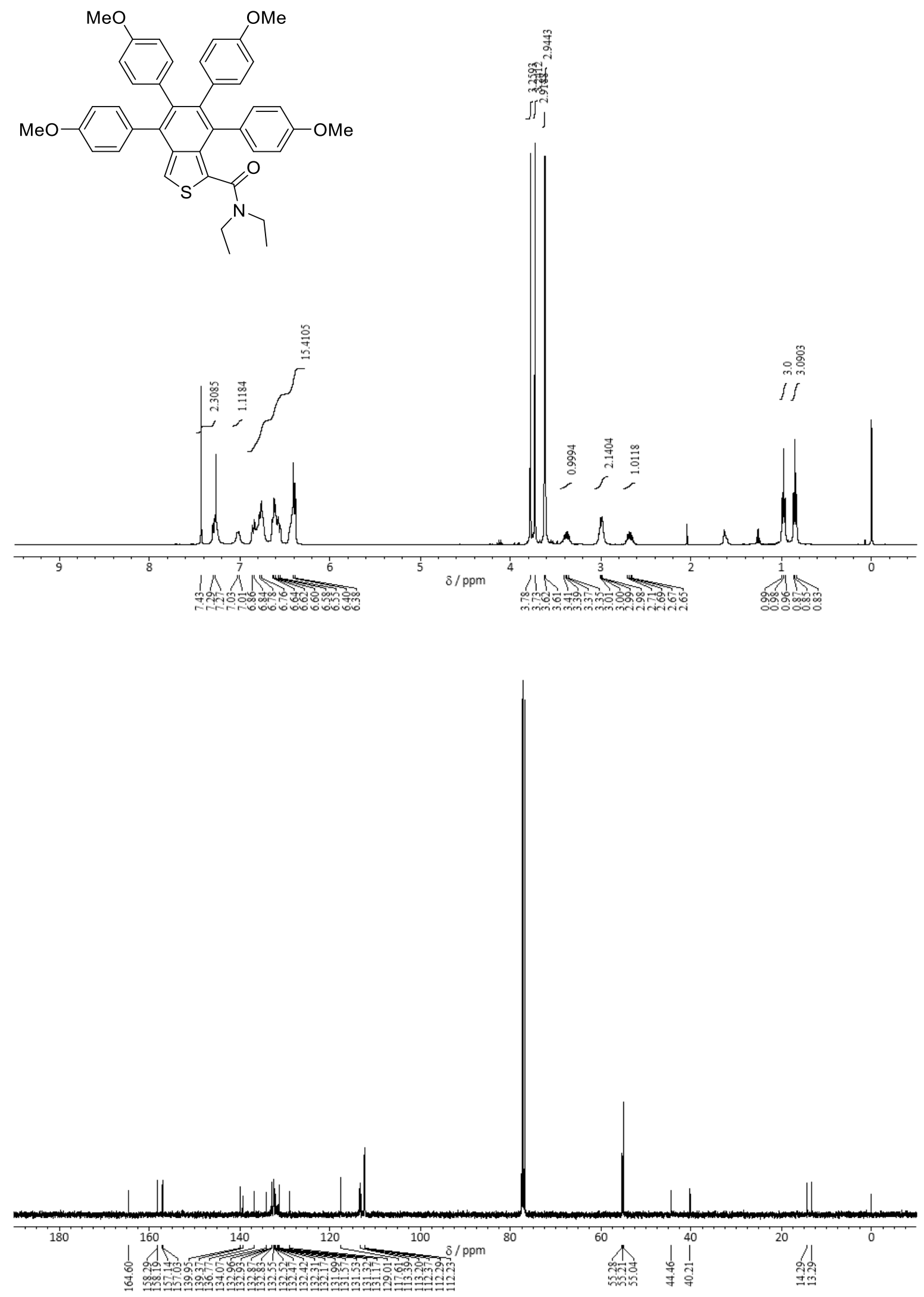
4,5,6,7-Tetrakis(3,4-dimethoxyphenyl)- $N, N$-diethylbenzo[c]thiophene-1-carboxamide (3ch)<smiles>CCN(CC)C(=O)c1scc2c(-c3ccc(OC)c(OC)c3)c(-c3ccc(OC)c(OC)c3)c(-c3ccc(OC)c(OC)c3)c(-c3ccc(OC)c(OC)c3)c12</smiles><smiles>CCCCC</smiles>
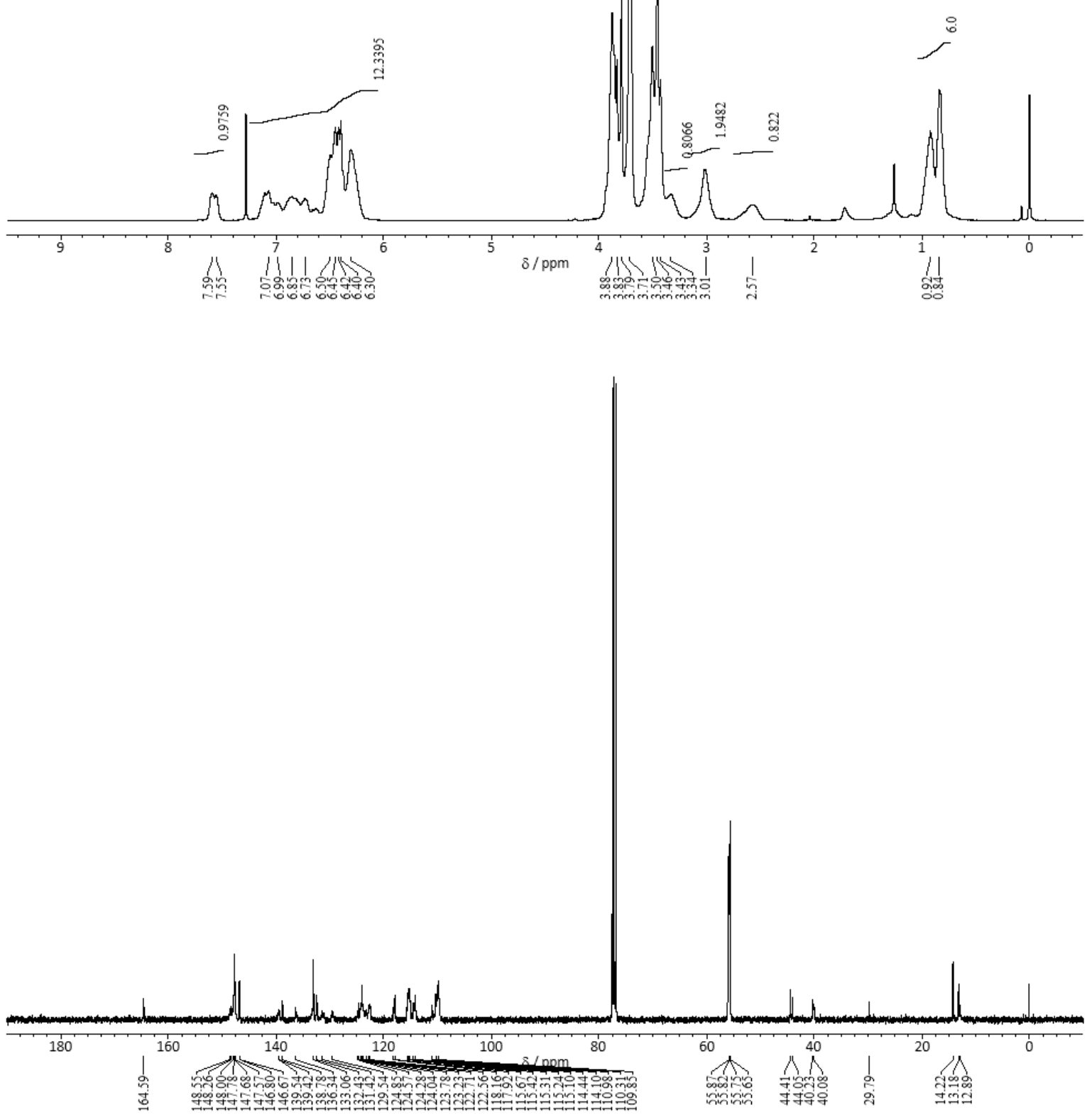
$N, N$-Diethyl-4,5,6,7-tetrapropylbenzo[c]thiophene-1-carboxamide (3ci)
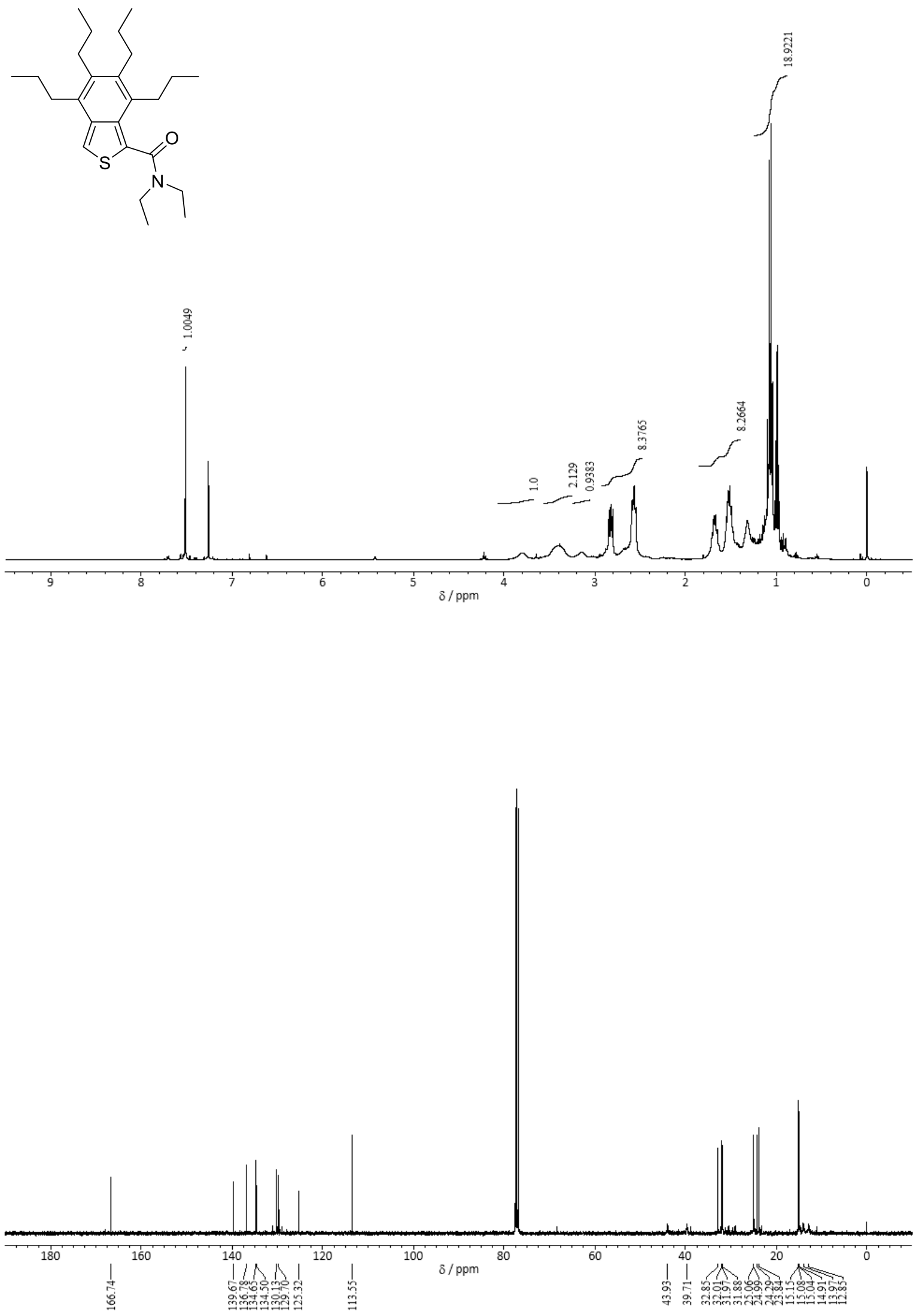
$N, N$-Diethyl-3-methyl-4,5,6,7-tetraphenylbenzo[c]thiophene-1-carboxamide (3ha)<smiles>CCN(CC)C(=O)c1sc(C)c2c(-c3ccccc3)c(-c3ccccc3)c(-c3ccccc3)c(-c3ccccc3)c12</smiles>
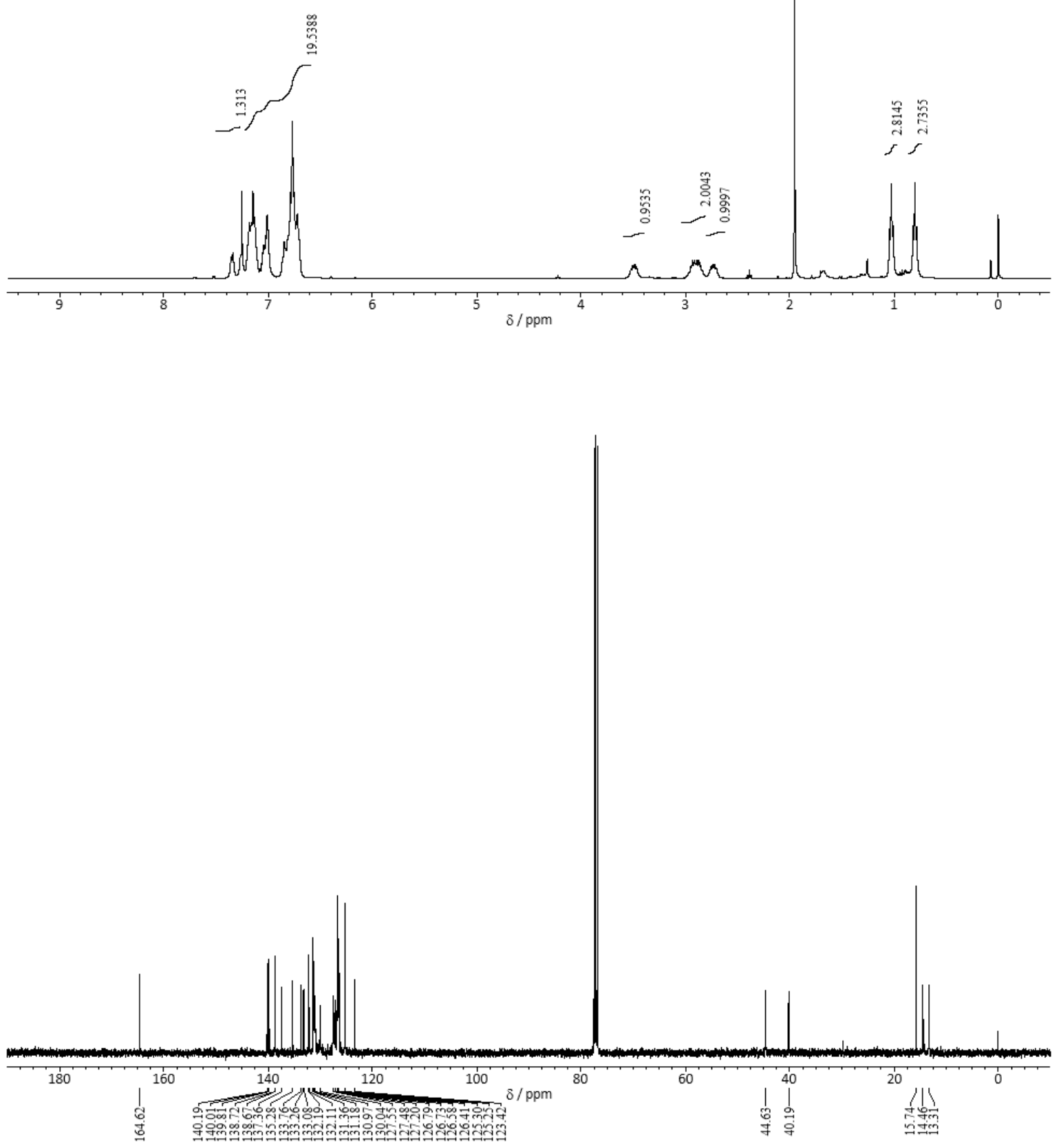
$N, N$-Diethyl-3,4,5,6,7-pentaphenylbenzo[c]thiophene-1-carboxamide (3ia)
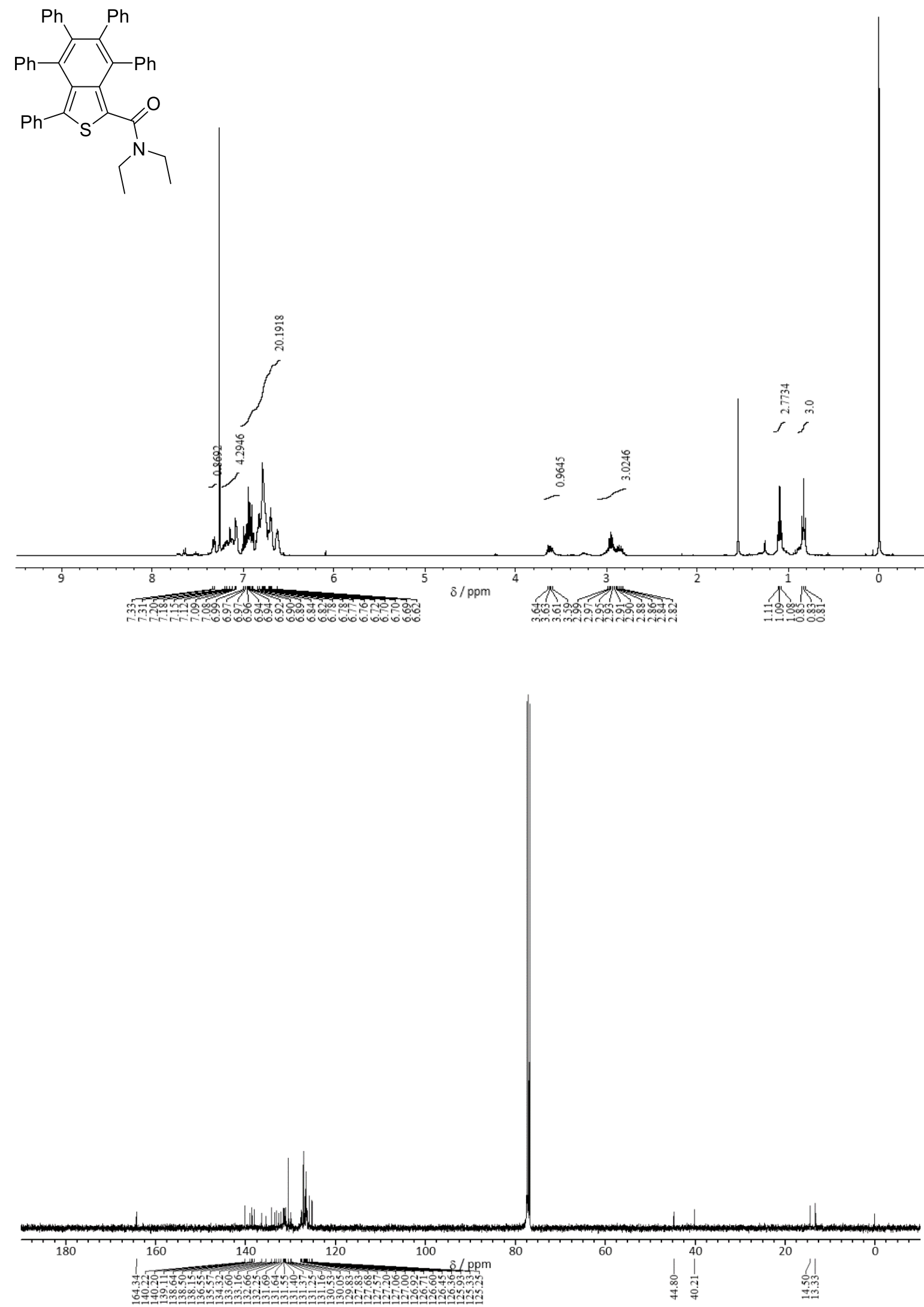
$N, N$-Diethyl-3-phenyl-4,5,6,7-tetrakis(4-(trifluoromethyl)phenyl)benzo[c]thiophene-1-carboxamide (3ie)
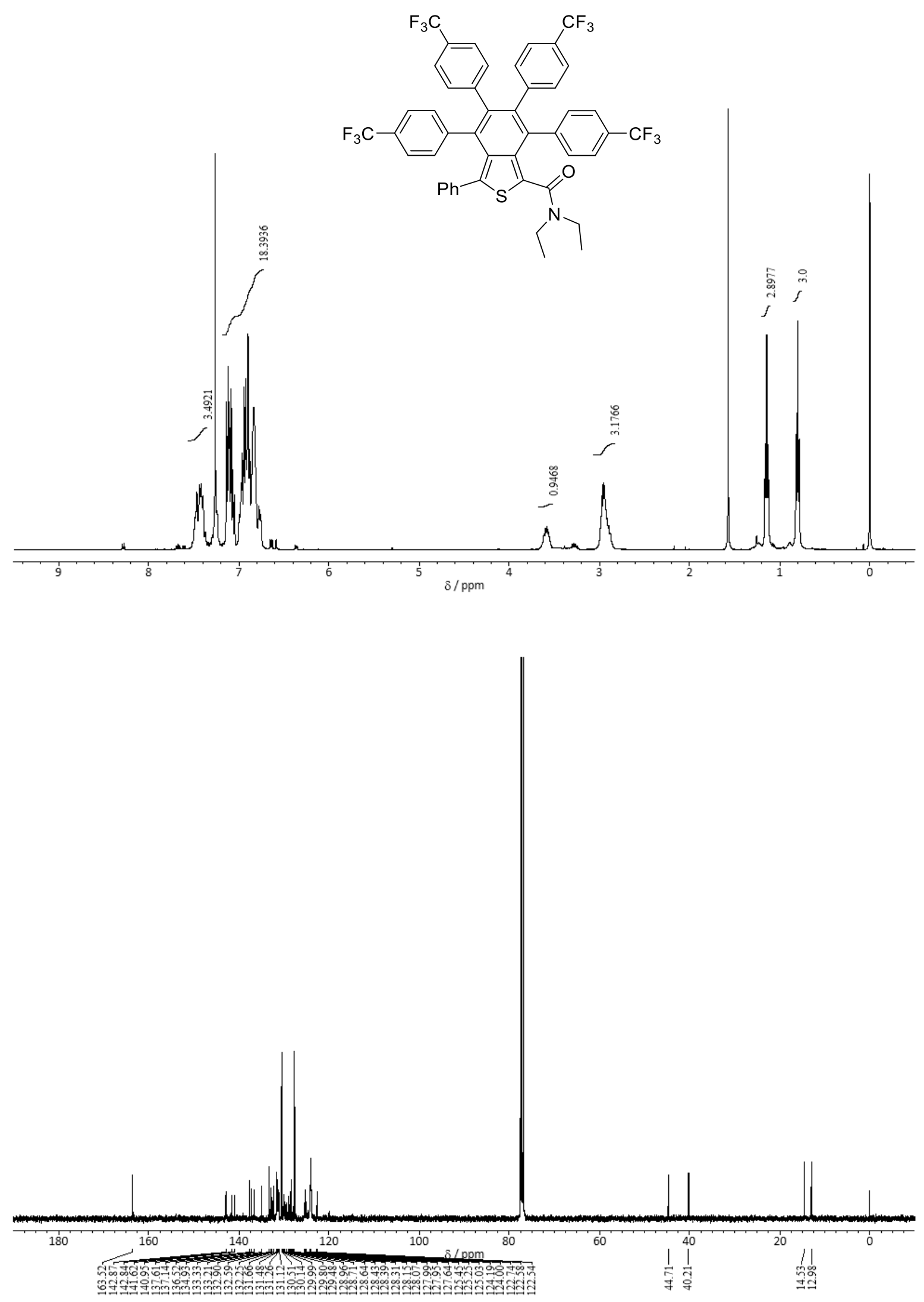
${ }^{13} \mathrm{C}\left\{{ }^{1} \mathrm{H},{ }^{19} \mathrm{~F}\right\}$ NMR spectrum for 3ie

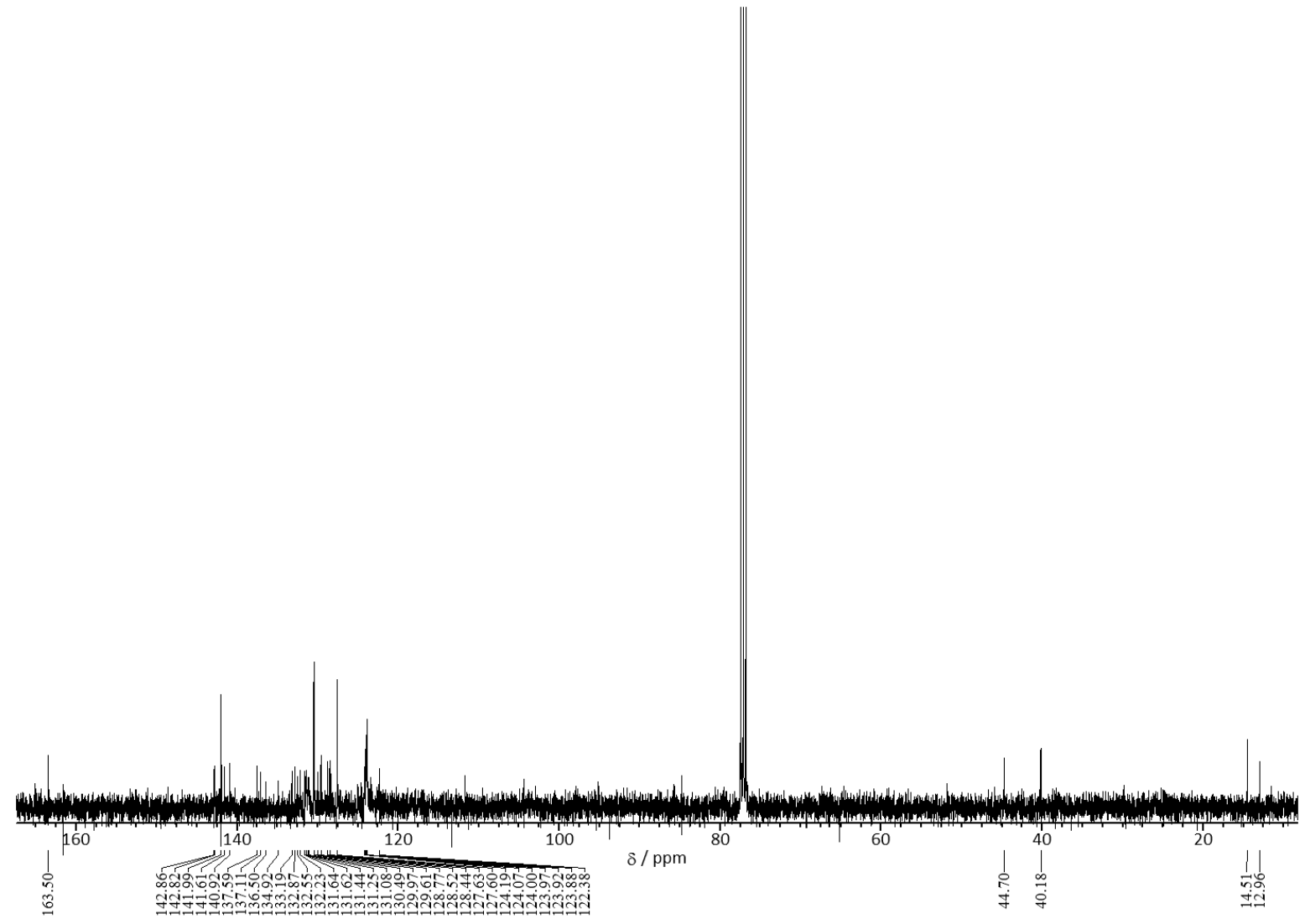


3-Chloro- $N, N$-diethyl-4,5,6,7-tetraphenylbenzo[ $c]$ thiophene-1-carboxamide (3ja)
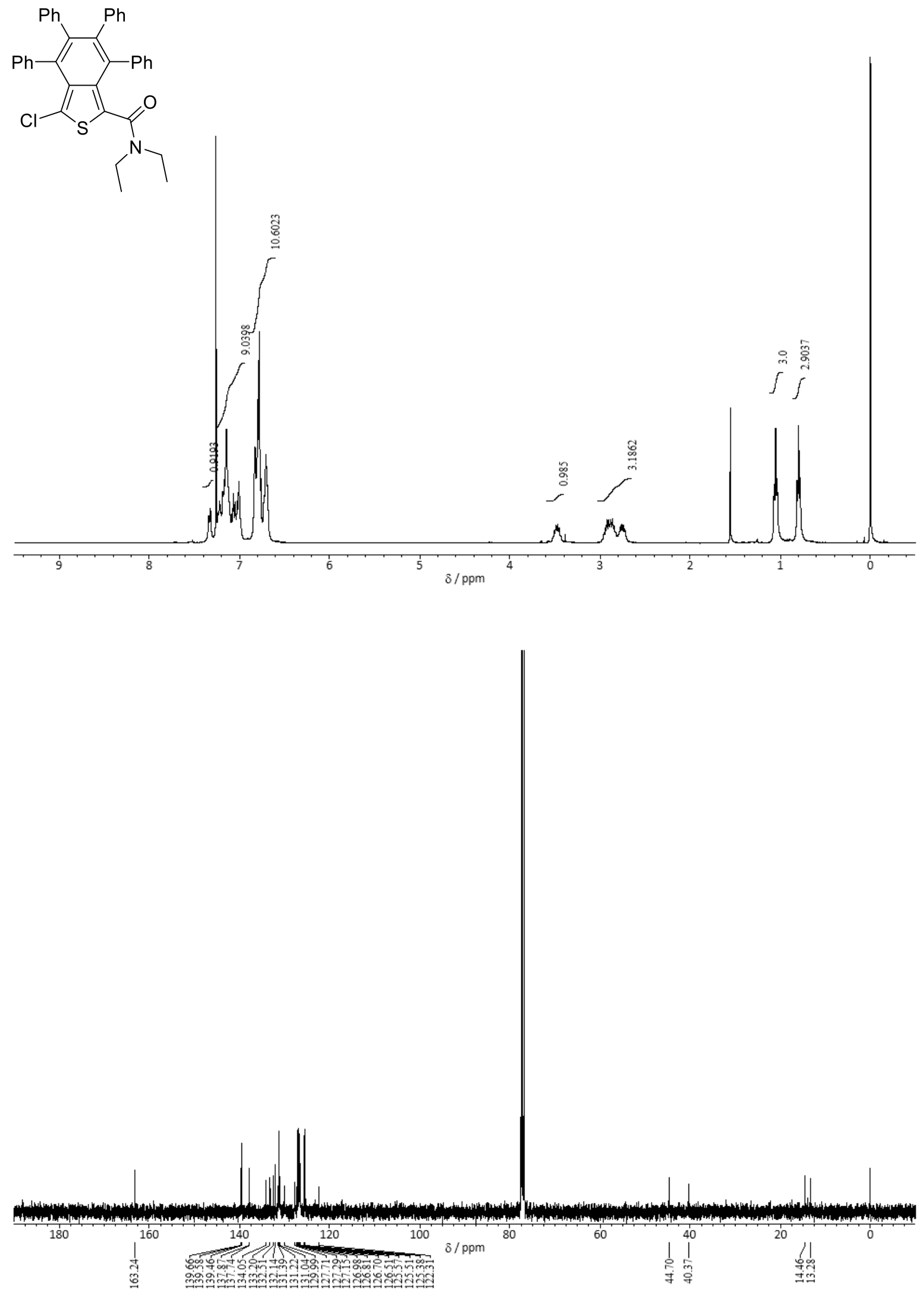
(E)-3-(1,2-Diphenylvinyl)- $N, N$-diethylthiophene-2-carboxamide (4ca)
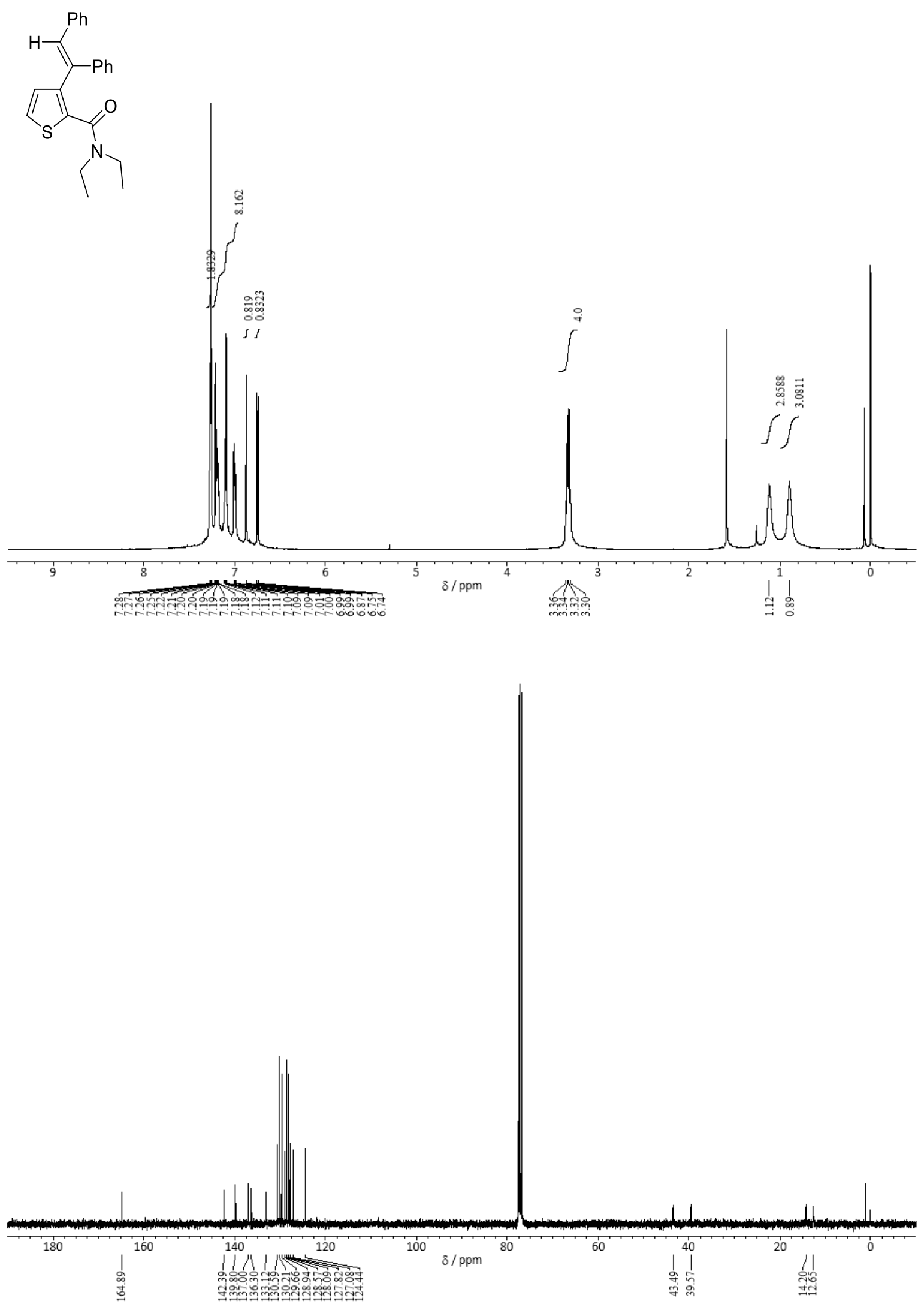


\section{${ }^{1} \mathrm{H}-{ }^{1} \mathrm{H}$ NOESY spectrum of $\mathbf{4} \mathbf{c a}$}

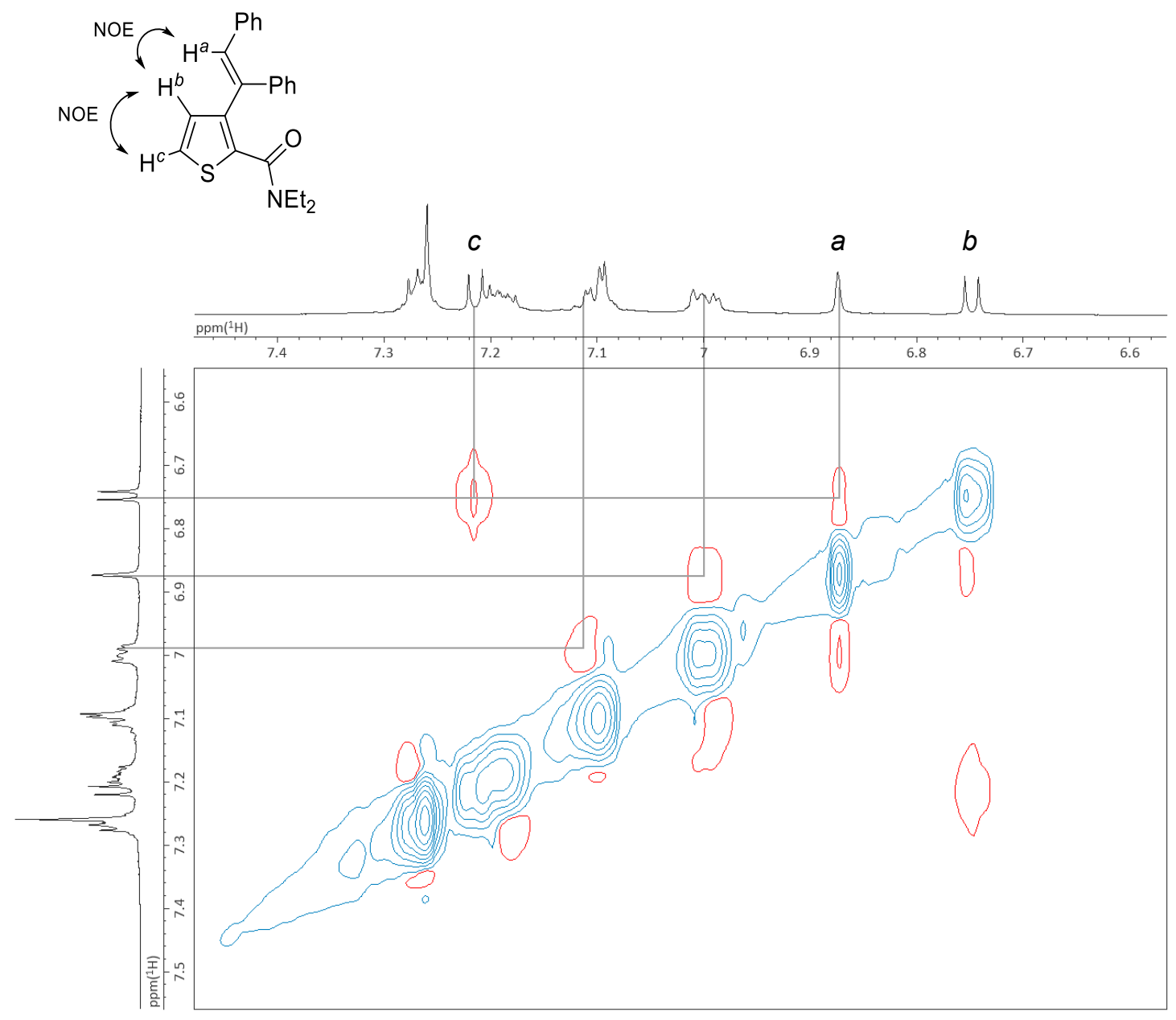


2-(4,5,6,7-Tetraphenylbenzo[c]thiophen-1-yl)pyridine (6)

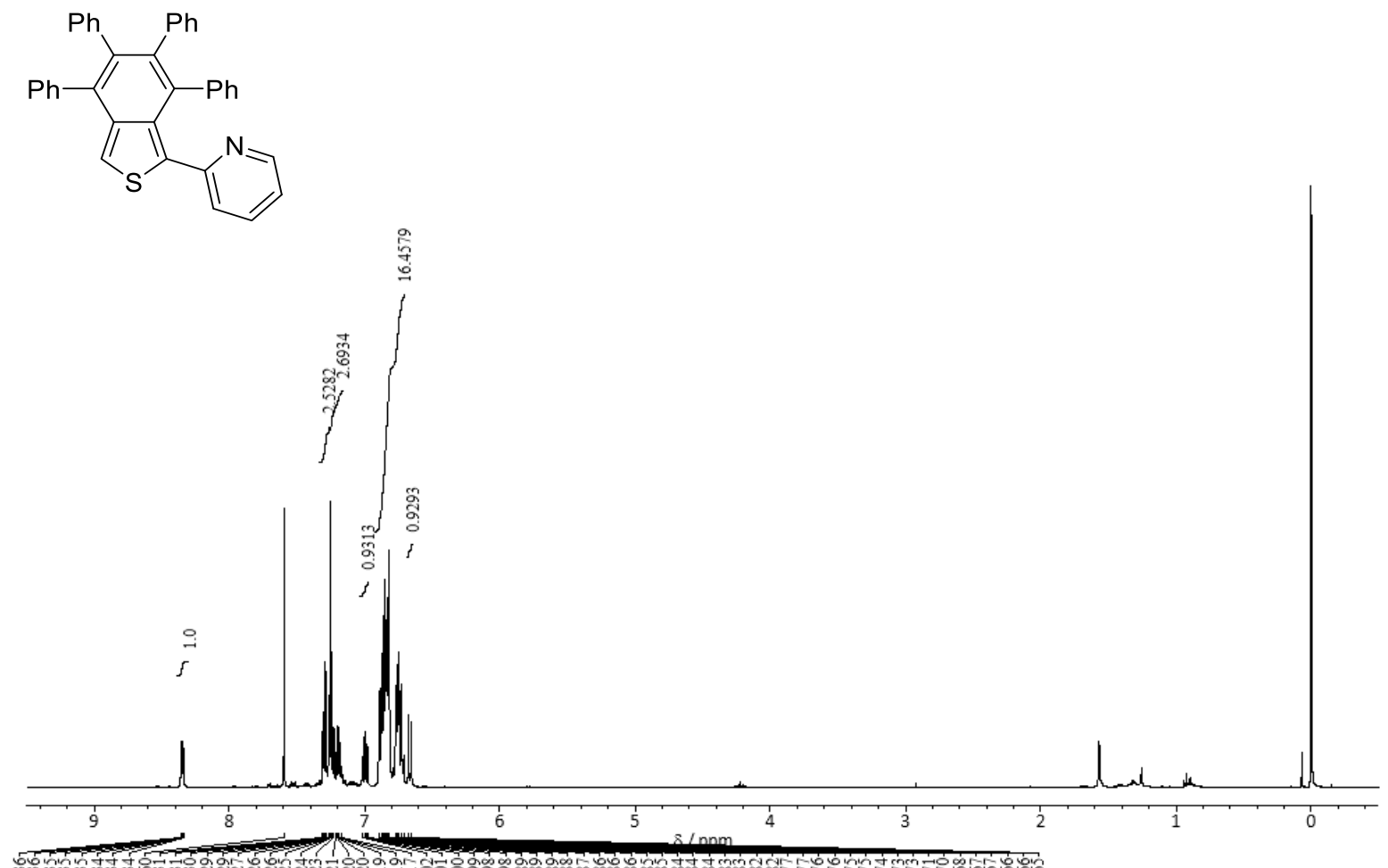

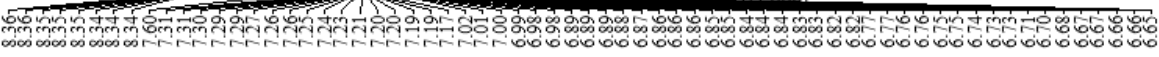

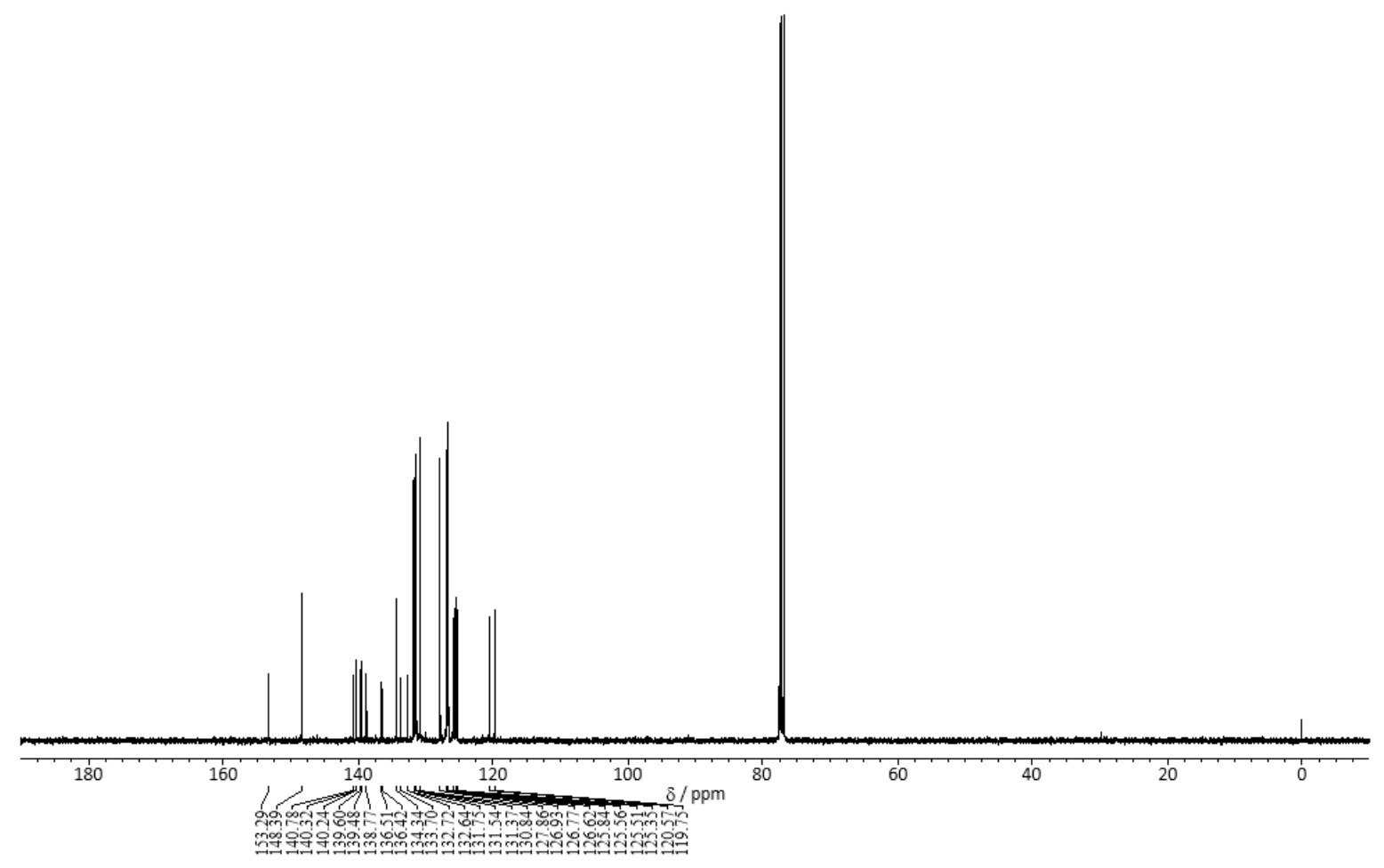


7-methyl-4-phenyl-5-(5,6,7,8-tetraphenylnaphthalen-1-yl)thieno[2,3-c]pyridine (8)

(Known compound: Liu, B.; Hu, F.; Shi, B.-F. Adv. Synth. Catal. 2014, 356, 2688-2696.)

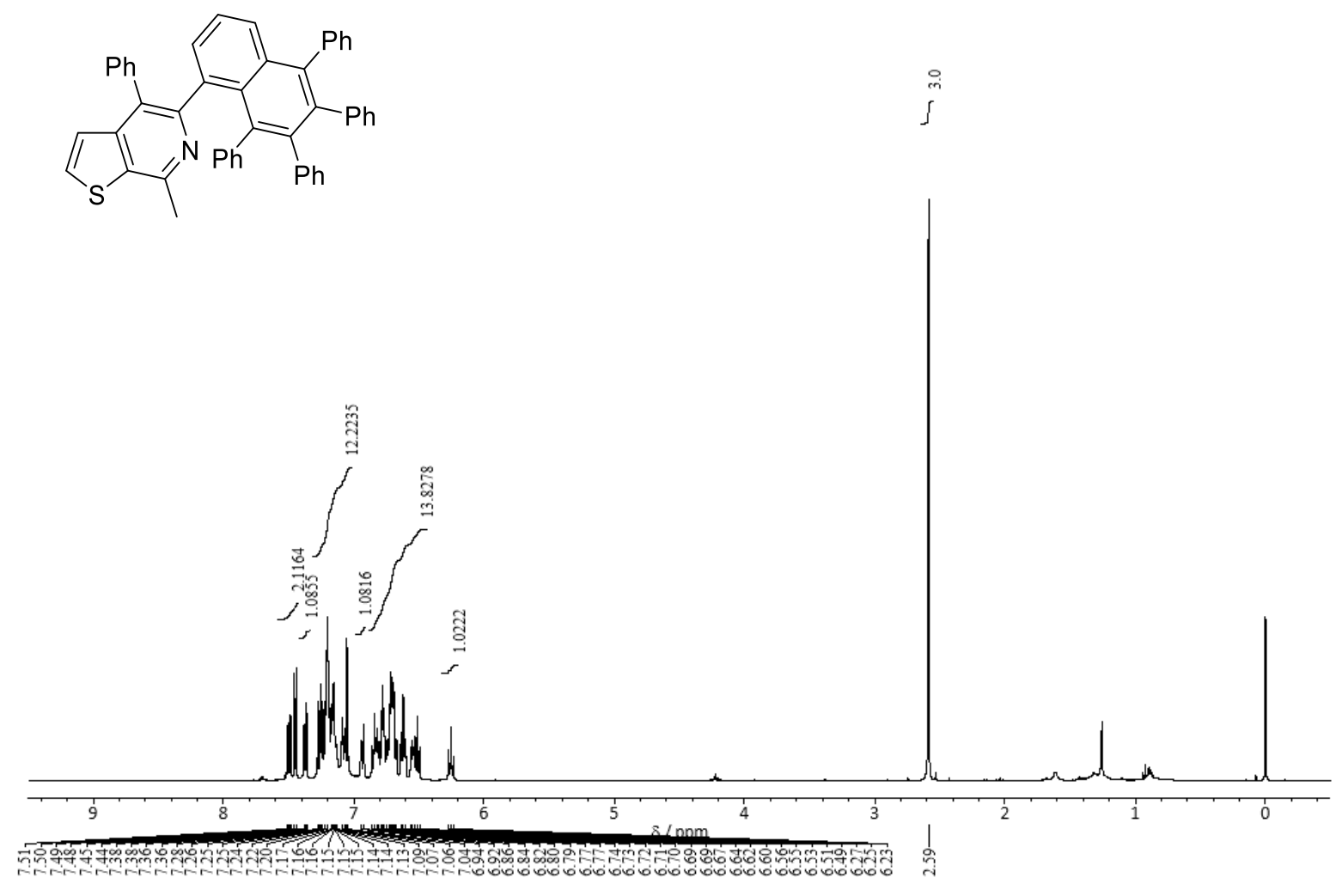


$N, N$-Diethyl-4,5,6,7-tetraphenyl-3-( $p$-tolyl)benzo[c]thiophene-1-carboxamide (9)
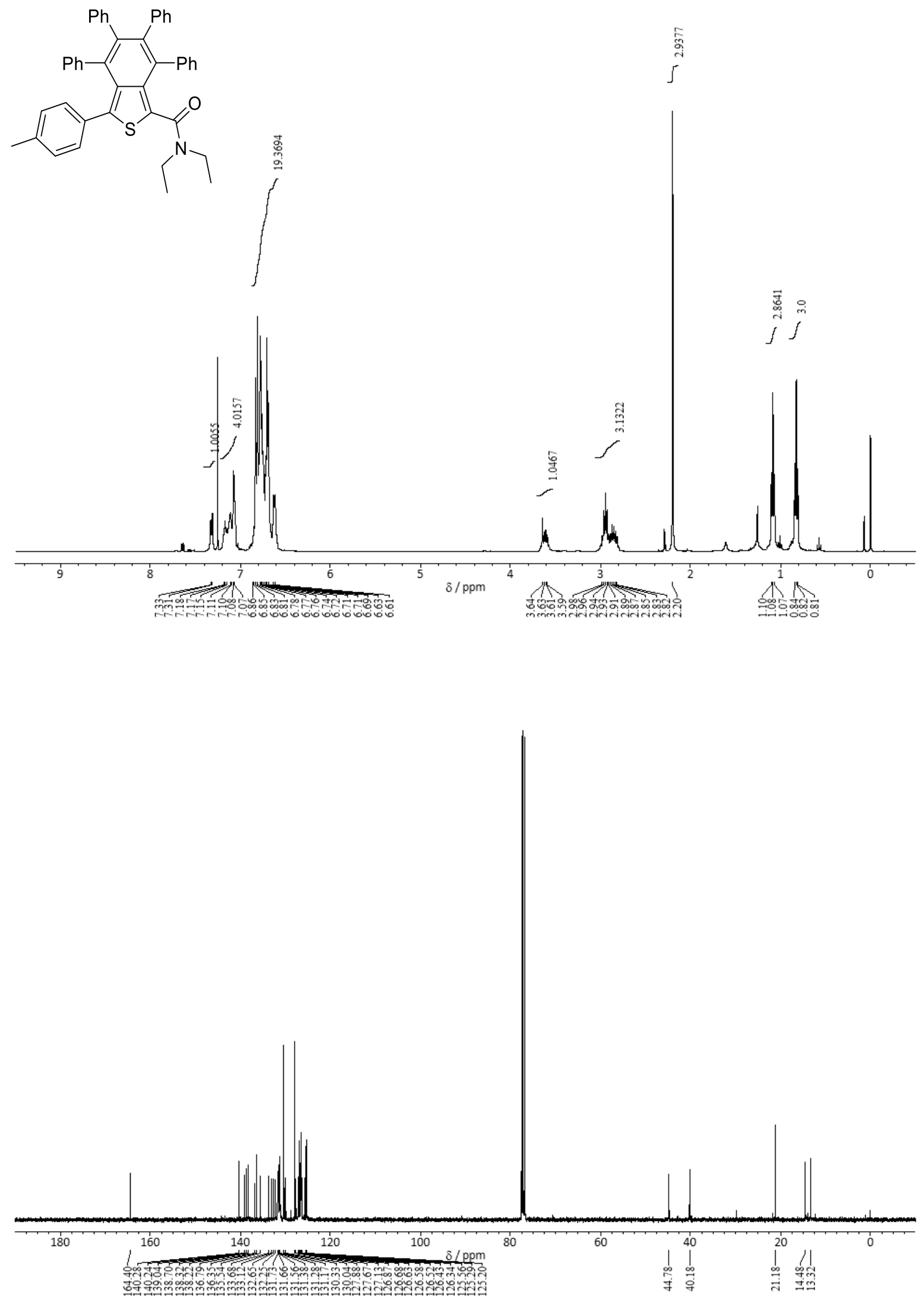